\title{
Effect evaluation of a Motivational Interviewing based counselling strategy in diabetes care.
}

Citation for published version (APA):

Heinrich, E., Candel, M. J., Schaper, N. C., \& de Vries, N. K. (2010). Effect evaluation of a Motivational Interviewing based counselling strategy in diabetes care. Diabetes Research and Clinical Practice, 90(3), 270-278. https://doi.org/10.1016/j.diabres.2010.09.012

Document status and date:

Published: 01/12/2010

DOI:

10.1016/j.diabres.2010.09.012

Document Version:

Publisher's PDF, also known as Version of record

Document license:

Taverne

\section{Please check the document version of this publication:}

- A submitted manuscript is the version of the article upon submission and before peer-review. There can be important differences between the submitted version and the official published version of record.

People interested in the research are advised to contact the author for the final version of the publication, or visit the DOI to the publisher's website.

- The final author version and the galley proof are versions of the publication after peer review.

- The final published version features the final layout of the paper including the volume, issue and page numbers.

Link to publication

\footnotetext{
General rights rights.

- You may freely distribute the URL identifying the publication in the public portal. please follow below link for the End User Agreement:

www.umlib.nl/taverne-license

Take down policy

If you believe that this document breaches copyright please contact us at:

repository@maastrichtuniversity.nl

providing details and we will investigate your claim.
}

Copyright and moral rights for the publications made accessible in the public portal are retained by the authors and/or other copyright owners and it is a condition of accessing publications that users recognise and abide by the legal requirements associated with these

- Users may download and print one copy of any publication from the public portal for the purpose of private study or research.

- You may not further distribute the material or use it for any profit-making activity or commercial gain

If the publication is distributed under the terms of Article $25 \mathrm{fa}$ of the Dutch Copyright Act, indicated by the "Taverne" license above, 


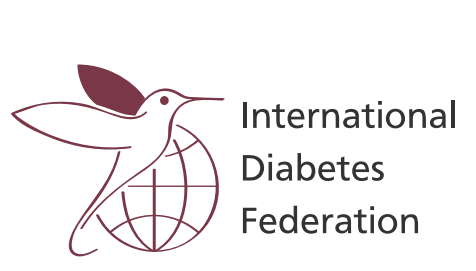

\title{
Effect evaluation of a Motivational Interviewing based counselling strategy in diabetes care
}

\author{
Evelien Heinrich $^{a, *}$, Math J.J.M. Candel ${ }^{b}$, Nicolaas C. Schaper ${ }^{c}$, Nanne K. de Vries ${ }^{a}$ \\ ${ }^{a}$ Department of Health Promotion, Maastricht University, Caphri, The Netherlands \\ ${ }^{\mathrm{b}}$ Department of Methodology and Statistics, Maastricht University, Caphri, The Netherlands \\ ${ }^{\mathrm{c}}$ Division Endocrinology, Department of Internal Medicine, University Hospital Maastricht, Caphri, The Netherlands
}

\section{A R T I C L E I N F O}

Article history:

Received 7 May 2010

Received in revised form

5 September 2010

Accepted 16 September 2010

Published on line 14 October 2010

Keywords:

Diabetes mellitus

Motivational Interviewing

Randomized controlled trial

Primary care

Behaviour change counselling

\begin{abstract}
A B S T R A C T
Aim: The present study assessed the effects of a Motivational Interviewing (MI) based counselling training for nurses on clinical, behavioural and process outcomes among diabetes type 2 patients.

Methods: The study is an RCT with follow-up measurements after 12 and 24 months. Thirtythree nurses and 584 patients participated. Nurses in the experimental condition received the training; control group nurses were trained after the study. The training consisted of two training sessions, two follow-up meetings, written feedback and three direct feedback sessions. Basic MI-principles and techniques and an MI-based counselling protocol were addressed.

Results: Results indicated disadvantageous effects on fat intake and HDL and advantageous effects on chance locus of control and knowledge. No effects were found on vegetable or fruit intake, physical activity, HbA1c, weight, blood pressure, total cholesterol, LDL, triglycerides, health care climate, quality of life or on self-efficacy.

Conclusions: As in other MI studies, mixed results were found. It would be premature to recommend dissemination of MI in diabetes care. More studies are needed in real-world settings with health care professionals of the field instead of intensively trained MI interventionists. Knowledge should be gained about adequate training and factors contributing to the implementation of MI in daily practice.
\end{abstract}

(C) 2010 Elsevier Ireland Ltd. All rights reserved.

\section{Introduction}

The incidence of diabetes type 2 (DM2) worldwide increases rapidly due to changing lifestyles and increased longevity [1,2]. In addition to physical and psychological burden to the patient, the increase in diabetes causes a financial burden to society [3]. Besides pharmacological treatment, lifestyle modification (such as adjustment of diet and physical activity) is a crucial element in treatment to prevent or delay the onset of complications [4-6]. Because of their daily responsibility for a large number of behavioural choices and activities, patients play a central role in their own treatment. Patients experience difficulties with self-management during daily life, contributing to frequent suboptimal control of risk factors [7-10]. To limit the consequences of the increase of patients with DM2, effective ways of patient counselling are urgently needed.

\footnotetext{
* Corresponding author at: Maastricht University, Department of Health Promotion, C/o E. Heinrich, P.O. Box 616, 6200 MD Maastricht, The Netherlands. Tel.: +31 043 3882422; fax: +31 0433671032 .

E-mail address: Evelien.Heinrich@gvo.unimaas.nl (E. Heinrich). 0168-8227/\$ - see front matter (C) 2010 Elsevier Ireland Ltd. All rights reserved. doi:10.1016/j.diabres.2010.09.012
} 
A promising counselling strategy in the treatment of lifestyle problems and disease is 'Motivational Interviewing' (MI). MI is defined as 'a client-centred, directive method for enhancing intrinsic motivation to change by exploring and resolving ambivalence' (p. 25) [11]. In contrast to traditional, more paternalistic, counselling styles, MI gives the patients' knowledge and experiences a central role in finding the best behaviour change strategies. The motivation to change should originate from the patient instead of being imposed by the health care professional.

MI was originally developed in the addiction field, but this relatively new counselling style is increasingly being advocated in other health areas such as diet, exercise, and diabetes [12-14]. Nevertheless, consistent evidence for the effectiveness of MI in these areas is still limited and additional research is indicated [14-16]. MI in chronic disease care may require a different approach than in addictive behaviours and the health care providers are different [14]. However, as summarized by Martins and colleagues [17], MI shows potential for diabetes care. In some of the nine studies reviewed by Martins and colleagues [17], beneficial effects on glucose levels, physical activity, weight and engagement in dietary changes were found. In all studies the intervention consisted of separate (additional) MI-sessions aimed at behaviour change instead of MI embedded in usual care.

In conclusion, MI seems promising for diabetes care but its effectiveness when incorporated in daily practice and not as a separate intervention in addition to usual care is still unclear. Therefore, the present study determined the effects of an MIbased counselling training to nurses on clinical, behavioural and process outcomes in DM2 patients. Nurses were supposed to use the counselling style during usual care.

\section{Methods}

\subsection{Study design and procedures}

In The Netherlands, diabetes care is provided mainly in primary care. In most practices patients are seen annually by their general practitioner and quarterly by a "practice" nurse, whose main tasks are monitoring the disease, providing education and lifestyle counselling. These nurses specialize in the care in chronic diseases such as diabetes and COPD, and are supervised by the general practitioner.

General practices were recruited in the southern parts of The Netherlands. In total 33 nurses participated. Nurses were asked to list all eligible patients within their practice(s): DM2 for $\leq 5$ years and an age between 40 and 70 years. Patients were excluded if they had severe co-morbidity or insufficient command of the Dutch language. All patients were invited by letter to participate in the study. Of the 1517 patients approached, 618 patients (41\%) signed informed consent. Based on the in- and exclusion criteria, 584 patients were included.

This study is a cluster randomized controlled trial. Nurses within a district frequently contact each other and have shared training sessions. To avoid contamination, cluster randomization was chosen. With a computerized randomizer, two districts (18 nurses) were assigned to the experimental condition and two districts (15 nurses) were assigned to the usual care condition. Nurses could not be blinded but assessment of outcomes was done by an independent observer.

The intervention started in autumn 2006, when nurses of the experimental condition were trained. The effects of the intervention were measured among patients. Besides a pretest before the start of the intervention, post-tests were conducted after 12 and 24 months (autumns of 2007 and 2008).

The medical ethics committee of the academic hospital Maastricht and Maastricht University approved the study.

\subsection{Intervention}

Nurses in the experimental condition received an MI-based counselling training after baseline measurements. Nurses were supposed to apply the new counselling style during standard quarterly consultations with their patients. Patients of control group nurses received usual care: quarterly consultations with a nurse who did not attend the MI-based training.

During the two years of study, nurses and patients from the experimental and control group had access to a web-based education programme (www.diep.info) that was developed to support self-management by patients. This programme does not only provide information, but also offers tools to support self-reflection, goal setting, problem solving, and active patient participation. The development and content of this programme is described in detail elsewhere [18].

\subsubsection{Training}

Two 5-h sessions were organized to train nurses in an adapted form of MI and the use of the education tool. The MI part was given by a certified trainer. The training consisted of presentations, demonstrations, role-playing and discussions. Nurses received a project folder with information about the study, training material (e.g. cases for role-playing), background information about MI including examples of dialogues between health care professionals and patients, and information about the education tool and how to use it. Furthermore, nurses received instruction charts specifying counselling techniques to support them during consultations. In the week between the two sessions, nurses were asked to read all the MI background information and the information about the education programme (diep.info). Three months after the training, nurses received written feedback on two audio-taped consultations. Besides, nurses were visited three times during their work for direct feedback (3 h per visit), approximately 6, 9 and 11 months after the training. The first time this was done by the MI-trained researcher, while the second and third visits were done by the MI-trained teaching nurse. Additionally, nurses had two opportunities to assemble an afternoon to share experiences and practice together; 8 months and 18 months after the training. In total, approximately $21.5 \mathrm{~h}$ counselling training was given, and $5 \mathrm{~h}$ optional group meetings.

\subsubsection{Adapted Motivational Interviewing}

The counselling intervention was based on MI [11] and health behaviour change counselling [19] and was designed to fit to 


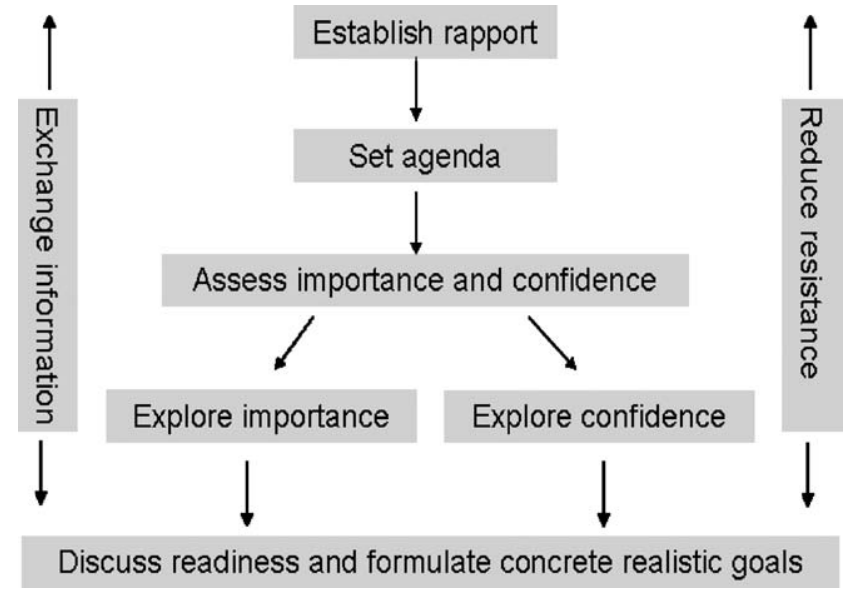

Fig. 1 - Counselling protocol.

diabetes care consultations (approximately 20 min per consultation).

During the first day of training, basic principles and techniques of Motivational Interviewing were addressed: expressing empathy, developing discrepancy, rolling with resistance, supporting self-efficacy, getting permission for actions, avoiding the provision of unsolicited advice and/or information, using open-ended questions instead of closed questions, using reflections and using summaries [11,19].

The second day focused on a MI-based counselling protocol, see Fig. 1, and the use of the education programme. The protocol consisted of the following steps: establish rapport, set agenda together with patients, assess importance and confidence, explore importance and confidence, summarize positive and negative issues of behaviour change, discuss a plan with patients, set concrete and realistic goals with a patient if the patient is ready for change and there is a need for goals, summarize the plan and ask if the patient agrees, and finish the consultation appropriately. Strategies that were learned to explore importance and confidence were the 0-10 strategy, the matrix of pros and cons, and brainstorming about solutions $[11,19]$. Counselling skills targeting behaviour change, for example exploring confidence, only had to be used when there was a reason to, like declining medical outcomes. Other skills such as basic principles and techniques should always be used.

\subsection{Outcome measures}

Measurements took place at baseline, after 12 months and after 24 months. Patients completed a self-administered, written questionnaire with mainly validated scales on selfmanagement behaviours and process-outcomes, supplemented with a question about the use of www.diep.info and background variables. The latter included age, sex, ethnicity, educational level, time since diagnosis and living situation (alone or together). Nurses recorded several clinical parameters.

\subsubsection{Self-management behaviours}

Fat, fruit and vegetable intakes were measured using validated food frequency questionnaires (FFQ) $[20,21]$. The fat FFQ assessed the frequency and amount of 19 products or product groups contributing most to saturated fat intake. Instead of grams fat, a fat score (range 0-80) is calculated because the FFQ does not cover all sources of saturated fat [20]. The FFQ for fruit and vegetable intake assessed the frequency and quantity of fruit, fruit juice and raw or cooked vegetables. Average fruit and vegetable intake in grams per day were calculated [21].

Two items of the Summary of Diabetes Self-Care Activities scale (SDSCA) were used to assess smoking (smoked during the past seven days; yes/no) and to assess whether the physical activity (PA) standard of $\geq 30$ min on at least 5 days a week was met (PA-norm) [22].

Additionally, to have a measure of the amount of PA and time spent sedentarily, the Dutch short form of the 'International Physical Activity Questionnaire' (IPAQ) was used [23,24]. This 'last 7 days recall form' assessed walking, moderateintensity activities, vigorous-intensity activities and sedentary time. For PA, a MET-min/week sum score was used as outcome measure (PA-score).

\subsubsection{Clinical parameters}

Nurses recorded the following clinical parameters up to 12 weeks old or late at baseline and up to 6 weeks old or late at follow-up: height and weight (for body mass index calculation), blood pressure (BP), glycated haemoglobin (HbA1c), fasting cholesterol (total, LDL, HDL) and triglycerides.

\subsubsection{Process outcomes}

According to the Self Determination Theory [25] increased perceived competence is influenced by perceived autonomy support and improves patient outcomes [26,27]. To asses the patient's perception of the degree to which the nurse supported autonomy, the Health Care Climate Questionnaire (HCCQ) was used (15 items, Cronbach's $\alpha=0.95$ ) [28]. It includes items such as "I feel that my physician has provided me with choices and options". Answers were given on a 1 (strongly disagree) to 7 (strongly agree) scale. Also, perceived competence was assessed by the Diabetes Management SelfEfficacy Scale for people with DM2 (DMSES) (18 items, Cronbach's $\alpha=0.81$ ) [29]. It includes items such as "I think I am able to overcome high blood glucose levels". The answering options range from 1 (no, certainly not) to 5 (yes, certainly).

Health Locus of Control (HLOC) was measured with the Dutch version of the diabetes specific health locus of control scale for diabetes patients [30]. Three loci of control were distinguished [31-33]: Internal, Powerful others and Chance HLOC referring to the beliefs that health outcomes are dependent on one's own behaviour (IHLOC, Cronbach's $\alpha=0.85$ ) [33], on the health care provider (PHLOC, Cronbach's $\alpha=0.75$ ) [33], or that health outcomes are random occurrences (CHLOC, Cronbach's $\alpha=0.72$ ) [33]. Each sub-scale consisted of six items with a Likert-scale ranging from 'fully agree' (1) to 'fully disagree' (6). Subscale scores were sum scores of all six items (range 6-36).

Diabetes specific quality of life (DSQoL) was measured with the Problem Areas in Diabetes Scale (PAID) (20 items, Cronbach's $\alpha=0.95)$ [34]. This is a self-report measure of diabetes-related distress. Respondents indicate the extent to 
which a specific aspect of diabetes management is a problem, on a 5-point Likert scale ranging from 'no problem' (0) to 'a serious problem' (4). For example, "Feel discouraged about your treatment".

Additionally, diabetes knowledge was measured by a multiple choice questionnaire (50 items), with a minimum of 0 (zero correct answers) and a maximum score of 50 (all 50 questions correct). To our knowledge there was no validated Dutch knowledge questionnaire, therefore we developed our own. The questionnaire addressed questions about diet (e.g. Does milk contain carbohydrates?), PA (e.g. Through regular exercise, blood glucose levels can increase), causes and consequences of diabetes (e.g. Diabetes can cause cardiovascular diseases), and overweight and diabetes (e.g. In patients with diabetes who are overweight, insulin works better than in patients with a healthy weight).

\subsection{Analyses}

Prior to our study, a power analysis (using Health Locus of Control as criterion variable) led to an initial sample size needed of 120 patients per cell $(p<.05$, power $=.09)$; because of expected drop-out we increased the sample size to the final 584 (also because sampling actually was done on the level of the nurses, not the patients).

Data analyses included descriptive statistics of the demographic variables and outcome parameters. Missing data on items of (sub)scales were replaced by the mean score of the individual on the other items of that (sub)scale, but only when no more than $20 \%$ of the values were missing. Scores on the DSQoL-scale and the PA-score were not distributed normally and therefore log-transformed. In the case of the HCCQ-score, which was also not normally distributed, transformation did not help. Therefore, next to the continuous HCCQ-outcome, a binary outcome measure based on the median (below or at the median versus above median) was computed. The same was done for the SDSCA-variable which measured the number of days an individual is physically active for at least $30 \mathrm{~min}$ a day. This variable was transformed into a binary variable for the analysis; 'A minimum of $30 \mathrm{~min}$ PA on at least 5 days a week: yes/no'.

Since we had multiple measurements and patients were nested within practices, multilevel analyses were conducted to test differences between the experimental and control group at baseline and at follow-up measurements regarding behavioural, clinical and process outcomes, correcting if necessary for influences of time since diagnosis, age, sex, educational level and living situation. Within some practices there was more than one nurse. Patients were not seen solely by one of them and it was impossible to trace which patient was seen by which nurse at all consultations. Therefore it was decided to omit nurses as a level from the analysis. Continuous outcome measures were analyzed with linear mixed regression in SPSS and binary outcome measures with logistic mixed regression in MLwiN [35]. In the multilevel approach available cases are included instead of only complete cases [36], thus enabling an intention-to-treat analysis. An analysis on available cases results in more statistical power. Furthermore, the multilevel approach assumes that missings are at random (MAR), that is, they may depend on variables included in the model. This is more flexible than assuming that missings are completely at random (MCAR), in which case they are not allowed to depend on any variable, an assumption made when performing the analyses only on complete cases.

Random slopes and random intercepts were tested first, followed by the fixed effects according to a top-down procedure. The least and non-significant components were deleted step-by-step from the model with a significance level of 0.10 . In the most reduced model, the intervention effects were examined with a 0.05 significance cut-off point. First an overall intervention effect across both follow-up measurements was tested. Only when the overall effect was significant, short and long term effects of the intervention were examined separately. In all analyses, a correction for baseline scores was made.

\section{Results}

\subsection{Descriptives}

From a total of 36 general practices, 584 patients started in our study of which 537 (92\%) filled out the baseline questionnaire, 447 (77\%) filled out the questionnaire at twelve months follow up and finally 423 (72\%) filled out the questionnaire at 24 months. Of all patients, 389 (67\%) filled out all three questionnaires and 32 (5\%) filled out none.

Concerning clinical parameters, $570(98 \%)$ patients had at least one valid value at baseline, 498 (85\%) at 12 months follow up and 462 (79\%) at 24 months.

The average age of the patients was 59 years $(S D=5.27)$ and somewhat less than half of them were female (44.9\%). Most patients had lower education (61.7\%), 23.6\% had a medium level of education and $14.7 \%$ had a high educational level. Approximately one quarter (26.4\%) of the patients was diagnosed with diabetes $\leq 1$ year, $47.0 \%$ between 2 and 3 years and $26.6 \% 4$ and 5 years. During the 2-year follow up, $138(29.1 \%)$ patients visited the educational programme (diep.info) at least once. In the control group, $79 \%$ of the patients never visited the website and another $10 \%$ only visited the website once. For the experimental group these percentages are $73 \%$ and $13 \%$ respectively. At baseline, $77.3 \%$ had an HbA1c level below 7\% (norm score); approximately 41.3\% had a (Body Mass Index) BMI below 28 and 42.2\% had a BMI above 30 .

Tables $1 \mathrm{a}$ and $1 \mathrm{~b}$ show baseline scores and the results of the comparison between experimental and control group. No significant differences between the experimental and control group were found in patient characteristics or outcome measures, except for smoking status, physical activity and fat intake. Patients of the experimental group had a lower fat intake (-1.5 points), a lower PA-score (-110 MET-min/week), and smoked more often $(+8.8 \%)$.

\subsection{Intervention effects}

Table 2 shows the corrected effect estimates for self-management behaviours (vegetable, fruit and fat intake, physical activity, sedentary time and smoking) after 12 months and after 
Table 1a - Baseline continuous outcome scores and differences between experimental and control group.

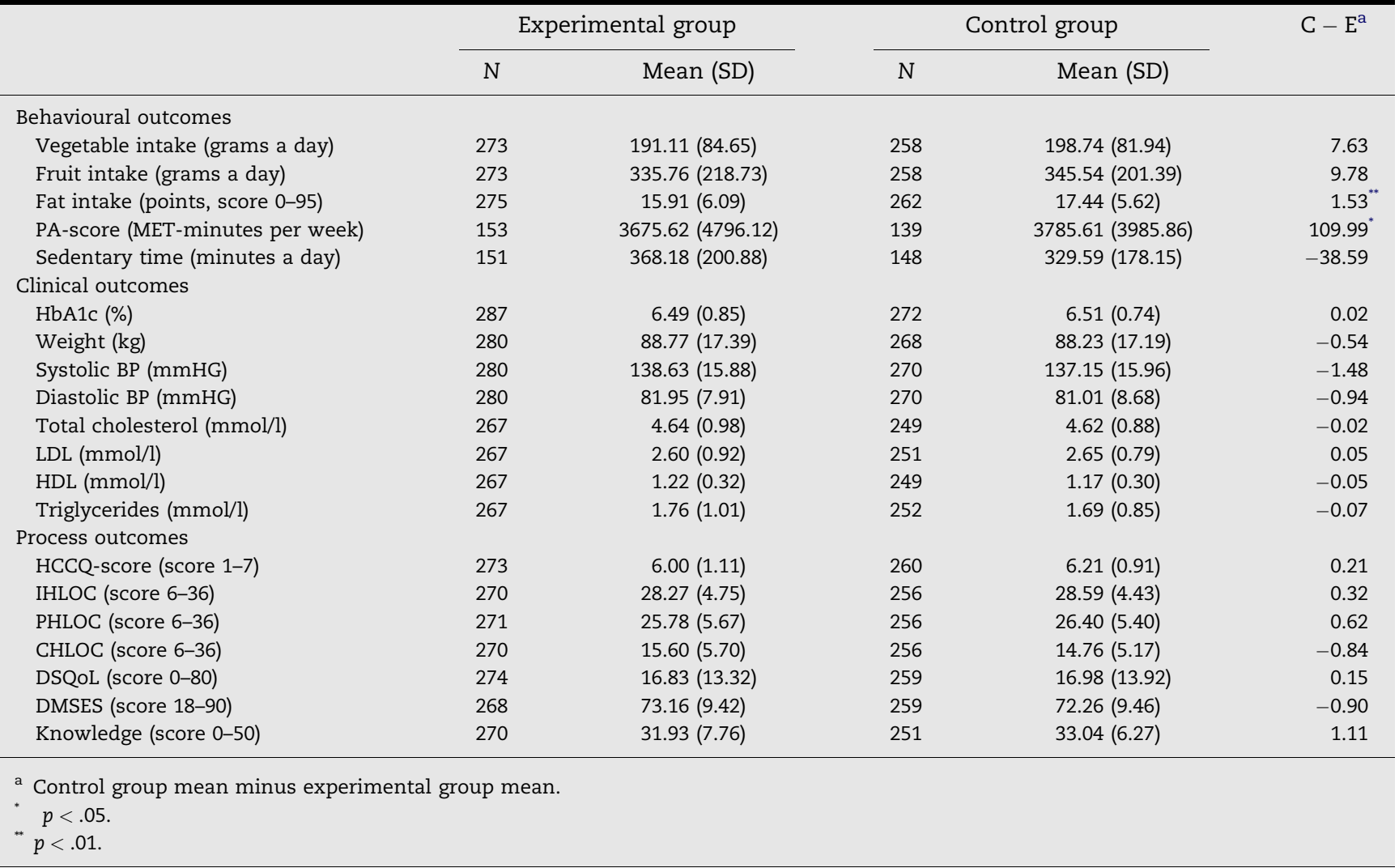

24 months follow-up. No significant differences between experimental and control group were found except for fat intake in favour of the control group. Correcting for baseline scores, patients of the experimental group had a higher fat intake both at 12 months follow-up and at 24 months follow-up $(p<0.05)$ when compared to the control group.

Concerning clinical parameters, at 12 months follow-up no intervention effects were found, see Table 3. At 24 months follow-up there is a significant difference between groups for HDL-cholesterol in favour of the control group. Compared to the control group, HDL-cholesterol was significantly lower in the experimental group at 24 months follow-up $(p<0.01)$.
The only significant intervention effects in favour of the experimental group were found on process outcomes, as shown in Table 2. On both follow-up measurements, 12 and 24 months, scores on the chance HLOC of the experimental group were significantly lower $(p<0.01)$ and their knowledge-scores were significantly higher (12 months; $p<0.01$, 24 months; $p<0.05)$ compared to the control group.

\section{Discussion}

The present study was an evaluation of an MI-based counselling strategy embedded in usual diabetes care. Results

Table 1b - Baseline binary outcome scores and differences between experimental and control group.

$$
\text { Experimental group: } N(\%) \quad \text { Control group: } N(\%)
$$

Behavioural outcomes

PA-norm:

Meets norm $165(61.1 \%)$

Does not meet norm 105 (38.9\%)

$154(59.7 \%)$

$104(40.3 \%)$

Smoking:

Yes $\quad 73(26.5 \%)$

$45(17.3 \%)$

No $202(73.5 \%) \quad 215(82.7 \%)$

Process outcomes

HCCQ:

$\begin{array}{lll}\leq \text { Median } & 137(50.2 \%) & 126(48.5 \%) \\ >\text { Median } & 136(49.8 \%) & 134(51.5 \%)\end{array}$

a Differences in proportions between experimental and control group.

$p<.05 ; " p<.01$. 
Table 2 - Results from the multilevel analyses of behavioural and process measures comparing experimental with control group.

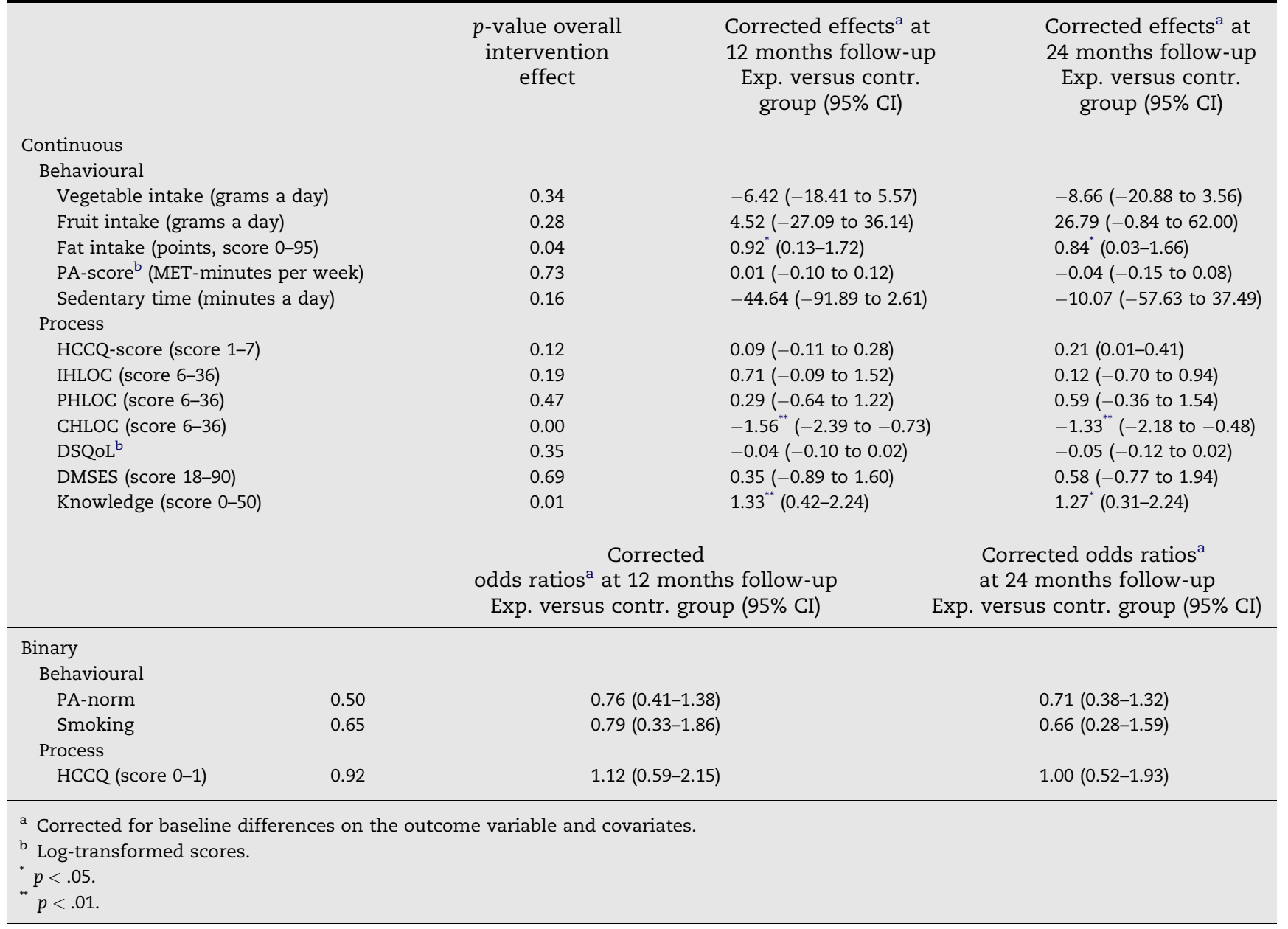

indicate no major intervention effects on outcome measures, although minor differences between groups were found at follow-up measurements for fat intake, HDL-cholesterol, chance health locus of control and knowledge.

Concerning fat intake and HDL-cholesterol an adverse effect was found. At baseline, the control group had a higher fat intake compared to the experimental group and a possible explanation for the decrease in fat intake in the control group could be that the subjects with a higher fat intake at baseline became more aware of their unhealthy behaviour as a result of filling out the baseline questionnaire. Alternatively, the differences at posttests might be a

Table 3 - Results from the multilevel analyses of clinical measures comparing experimental with control group.

$\begin{array}{cc}p \text {-value overall } & \text { Corrected effects }^{a} \\ \text { intervention } & \text { at } 12 \text { months follow-up } \\ \text { effect } & \text { Exp. versus contr. group }\end{array}$

(95\% CI)
Corrected effects ${ }^{\mathrm{a}}$ at 24 months follow-up Exp. versus contr. group $(95 \%$ CI)

$0.09(-0.05$ to 0.23$)$

$0.33(-0.57$ to 1.23$)$

$-1.29(-4.17$ to 1.59$)$

$1.27(-0.32$ to 2.86$)$

$-0.08(-0.56$ to 0.10$)$

$0.03(-0.13$ to 0.19$)$

$-0.07^{* *}(-0.12$ to -0.03$)$

$-0.02(-0.17$ to 0.14$)$

Triglycerides $(\mathrm{mmol} / \mathrm{l})$

$-0.02(-0.06$ to 0.01$)$

$-0.02(-0.16$ to 0.13$)$

a Corrected for baseline differences on the outcome variable and covariates.

$p<.05$.

$p<.01$. 
simple case of regression to the mean. Although an intervention effect was found on HDL-cholesterol, no effects were found on total or LDL-cholesterol. Besides, the corrected effect of $-0.07 \mathrm{mmol} / \mathrm{l}$ is so small that it might be seen as clinically irrelevant.

Advantageous effects were found on chance HLOC (CHLOC) and knowledge. The effect on CHLOC indicates that people in the experimental group were less inclined than the control group to relate their diabetes control to chance factors. Empowered patients are considered to perceive more control over their diabetes, which has been associated with better selfmanagement outcomes [37,38]. However, we found a decrease in CHLOC, but we did not find an increase in internal HLOC nor did we find a change in powerful others HLOC. Concluding that our intervention contributed to patient empowerment would therefore be premature and also inconsistent with the lack of effect on self-management outcomes. Concerning knowledge, due to the intervention patients might have had a more active role during consultations. As a result, patients possibly felt more confident to ask questions and this might have improved knowledge. However, the corrected effects of 1.33 and 1.27 mean a difference of $<1.5$ points on a scale of $0-50$ : a rather modest effect in terms of absolute magnitude.

All in all, based on our study we cannot conclude that our MI-based counselling strategy offers advantages over usual care. Previous research of MI in diabetes care is limited and has shown mixed effects. In two studies where MI-sessions given by a MI-trained psychologist were added to a weight loss intervention for female DM2 patients, effects of MI were found on glucose control $[39,40]$, on blood glucose monitoring [39] and on weight [40]. In another study where three individual MI sessions and three follow-up telephone calls were added to usual DM care, no effects were found on glucose control or $\mathrm{BMI}$, and inconsistent results were found for self-management behaviours [41,42]. Ismail and colleagues [43] evaluated four MI-sessions given by a diabetes nurse and added to usual care. They did not find any effect on glucose control, BMI, QoL or on self-management behaviours [43].

There are several factors in our study that might have influenced the outcomes. First of all: the interventionist. One of the differences between our study and previous studies is that our intervention was delivered by trained nurses instead of a psychologist. Although Rubak and colleagues [44] concluded that effectiveness was not related to the counsellor's educational background as medical doctor or psychologist, they did find that only 5 out of 11 studies that involved other health workers as MI-counsellors (e.g. nurses) were effective [44]. Perhaps we would have found better results when patients consulted a trained psychologist instead of a trained nurse. However, we aimed to embed MI in usual care, by usual health professionals.

A second factor that might have influenced the outcomes is that the counselling strategy had to be applied during regular consultations instead of separate MI-sessions in addition to usual care. This is especially difficult due to the different agendas of patients and nurses. Nurses' agendas may be influenced by treatment protocols and/or by reimbursement systems [45]. In usual diabetes care, not all consultations are dedicated to self-management. Self-management (including lifestyle changes) is only one topic besides other topics such as discussing side effects of medication, insurance issues, and eye examinations. In a study where MI was embedded in diabetes care, outcomes of consultations with a MI-trained dietician were compared to outcomes of consultations with a non-MI-trained dietician. As in our study, limited intervention effects were found. No effects were found on $\mathrm{HbA1c}$, BMI, fruit and vegetable consumption except on fat consumption [46]. Accordingly, more studies are needed to know what the implications are of MI embedded in diabetes care delivered by patients' usual care givers.

A third study characteristic that might have influenced the outcomes is the intervention delivery dose [14]. If nurses were unable to adequately implement the strategy, there is a risk of a type III error [47]. This possible risk should be taken into account when interpreting the findings of our study. Training nurses to change their behaviour might be as challenging as helping patients to change [48]. Perhaps the nurses should have been trained more intensively to practice the MI-based counselling strategy in daily work. We offered two training sessions, two follow-up meetings, written feedback once and three direct feedback sessions during daily work which is more than what is done in other studies where courses often have a workshop format without any kind of follow-up [49]. The question is how realistic it is to demand even more training time than what we did. A possible solution to this would be to incorporate training in MI counselling strategies during regular courses for students. Training intensity is only one aspect of the delivery dose. Also the total time spent on self-management changes during our intervention period may have been too limited. In accordance with the Dutch diabetes care guidelines, the patients in our study had 3-4 consultations per year in which all aspects of diabetes treatment had to be addressed. Previous MI studies in diabetes care offer specific MI sessions to patients which automatically results in more exposure time [17]. Review studies have shown that even small doses of MI ( $<15 \mathrm{~min})$ can be effective [44] but that higher treatment doses (>60 min) tend to increase the effectiveness [15,44]. Our intervention should be considered as low intensity MI and perhaps more consultations including MI-counselling were needed to be effective. Additional research is needed in this area as the effect of delivery dose is still inconclusive [17].

Fourth, the intervention content might have played an important role. We did not implement MI as it was originally developed in the field of substance use. We created an adapted form (AMI) suitable to quarterly consultations in DM care. However, a previous review and a meta-analysis specifically aimed at AMI-interventions showed beneficial effects $[15,16]$. Of course, our adaptation may have been suboptimal although we based it on earlier experiences.

A final study characteristic that might have been of importance is our patient population. We did not specifically aim to include people with suboptimal outcomes and looking at the clinical outcomes and some behavioural outcomes our populations seems relatively healthy. Approximately three quarters had a baseline HbA1c of $<7 \%$, the average systolic blood pressure was below $140 \mathrm{mmHG}$ and lipid profiles were near normal as well. Furthermore, self-reported vegetable and fruit intake were sufficient at baseline and $60 \%$ of the population reported to meet the physical activity norm. Also, 
the average baseline score on the measure for autonomy support was 6.1 on a scale of 1-7. According to the selfdetermination theory, autonomy supportive health care providers can influence a patient's autonomous motivation and thereby improve health outcomes [27]. However, at baseline patients already perceived their health care provider as autonomy supportive, indicating little improvement to expect. These data show a possible ceiling effect; little improvement may be expected from the intervention. On the other hand, more than $50 \%$ had a baseline BMI $>28$, and $40 \%$ did not meet the PA-norm, leaving ample room for improvement. Also, improvement of health locus of control dimensions, quality of life and self-efficacy could be made. Additional analyses to test the effects of baseline Hba1c and BMI on outcomes did not show significant interactions between intervention effects and baseline values. The apparent healthy baseline outcomes may be influenced by medication effects on clinical outcomes, which we unfortunately did not measure, and social desirability effects on dietary intake and PA. For example, it is known that fruit and vegetable intake is insufficient among Dutch adults [50]. Although a ceiling effect should be taken into consideration when interpreting our findings, it cannot fully explain the lack of effectiveness. Another population characteristic is our sample size but a lack of power seems unlikely when we compare our sample size to other studies [17,51].

Our results indicate a need for further research towards MIbased counselling embedded in usual care and assessment of factors influencing the use of such counselling strategies for a better understanding of the applicability of MI-interventions in diabetes care. Evaluations addressing these issues and a description of training methods are often lacking [16,48,52]. We gave a comprehensive description of the training and intervention. In addition to the current study, we evaluated tape-recorded consultations. These tapes show that some skills were implemented (e.g. agenda setting and assessing importance and confidence) but others were not (e.g. reflective listening and exploring importance and confidence). The implementation study also showed the importance of direct feedback on nurses' performances in daily practice and this study will be reported in the near future.

The present study had its limitations. There are no data available of patients or nurses who refused to participate. Therefore, we may have had a selection of nurses who have a special interest in new counselling techniques, which would limit external validity. However, with less motivated nurses it would have been even harder to find intervention effects. Furthermore, to avoid contamination we randomized on district level rather than on practice or patient level. Therefore, we corrected for possibly relevant baseline differences in all analyses. Additionally, we were not in control over counselling behaviours of control group nurses. For example, nurses were allowed to use the web-based education programme, but this limitation is inherent in the practicebased character of our study. Finally, behavioural outcomes were mostly measured by self-report questionnaires, which may have caused bias [53], but analyses of more objective clinical outcomes resulted in similar conclusions.

In sum, based on our study and what is known from other studies so far, it would be premature to recommend dissemination of MI in daily practice of diabetes care. More studies are needed in real-world settings with health care professionals of the field instead of intensively trained MI interventionists. Knowledge should be gained about adequate training and skill levels of health care professionals as well as about factors contributing to the implementation level in daily practice, such as characteristics of the general practices, patient populations, MI, and the health professionals themselves.

\section{Acknowledgement}

The study was supported by a grant from the Dr. Paul Janssen Foundation.

\section{Conflict of interest}

There are no conflicts of interest.

\section{R E F E R E N C E S}

[1] Wild S, Roglic G, Green A, Sicree R, King H. Global prevalence of diabetes, estimates for the year 2000 and projections for 2030. Diabetes Care 2004;27:1047-53.

[2] Deakin T, McShane CE, Cade JE, Williams RDRR. Group based training for self-management strategies in people with type 2 diabetes mellitus. Cochrane Database Syst Rev 2005.

[3] IDF (International Diabetes Federation). Diabetes atlas. 2nd ed. www.eatlas.ifd.org; 2008 [accessed 14.10.08].

[4] Adler AI, Stratton IM, Neil HA, Yudkin JS, Matthews DR, Cull CA, et al. Association of systolic blood pressure with macrovascular and microvascular complications of type 2 diabetes (UKPDS 36): prospective observational study. BMJ 2000;321:412-9.

[5] Norris SL, Lau J, Smith SJ, Schmid CH, Engelgau MM. Selfmanagement education for adults with type 2 diabetes: a meta-analysis of the effect on glycemic control. Diabetes Care 2002;25:1159-71.

[6] UK Prospective Diabetes Study Group. Intensive bloodglucose control with sulphonylureas or insulin compared with conventional treatment and risk of complications in patients with type 2 diabetes (UKPDS 33). Lancet 1998;352:837-53.

[7] Clement S. Diabetes self-management education. Diabetes Care 1995;18:1204-14.

[8] Harris MI. Health care and health status and outcomes for patients with type 2 diabetes. Diabetes Care 2000;23:754-8.

[9] Jerant AF, Von Friederichs Fitzwater MM, Moore M. Patients' perceived barriers to active self-management of chronic conditions. Patient Educ Couns 2005;57:300-7.

[10] Vermeire E, Wens J, Van Royen P, Biot Y, Hearnshaw H, Lindenmeyer A. Interventions for improving adherence to treatment recommendations in people with type 2 diabetes mellitus. Cochrane Database Syst Rev 2005. Art. No.: CD003638. doi:10.1002/14651858.CD003638.pub2.

[11] Miller WR, Rollnick S. Motivational interviewing: preparing people for change. New York, NY: Guilford; 2002.

[12] Hettema J, Steele J, Miller WR. Motivational interviewing. Annu Rev Clin Psychol 2005;1:91-111.

[13] Miller WR, Rose GS. Toward a theory of motivational interviewing. Am Psychol 2009;64:527-37. 
[14] Resnicow K, DiIorio C, Soet JE, Ernst D, Borrelli B, Hecht J. Motivational interviewing in health promotion: it sounds like something is changing. Health Psychol 2002;21:444-51.

[15] Burke BL, Arkowitz H, Menchola M. The efficacy of motivational interviewing: a meta-analysis of controlled clinical trials. J Consult Clin Psychol 2003;71:843-61.

[16] Dunn C, Deroo L, Rivara FP. The use of brief interventions adapted from motivational interviewing across behavioral domains: a systematic review. Addiction 2001;96:1725-42.

[17] Martins RK, McNeil DW. Review of motivational interviewing in promoting health behaviors. Clin Psychol Rev 2009;29:283-93.

[18] Heinrich E, Schaper NC, De Vries NK. Development of the web-based type 2 diabetes education programme: DIEP. Eur Diabetes Nurs 2009;6:51-6.

[19] Rollnick S, Mason P, Butler C. Health behavior change. A guide for practitioners. London: Churchill Livingstone; 1999

[20] Van Assema P, Brug J, Ronda G, Steenhuis I. The relative validity of a short Dutch questionnaire as a means to categorize adults and adolescents to total and saturated fat intake. J Hum Nutr Diet 2001;14:377-90.

[21] Van Assema P, Brug J, Ronda G, Steenhuis I, Oenema A. A short dutch questionnaire to measure fruit and vegetable intake: relative validity among adults and adolescents. Nutr Health 2002;16:85-106.

[22] Toobert DJ, Hampson SE, Glasgow RE. The summary of diabetes self-care activities measure: results from 7 studies and a revised scale. Diabetes Care 2000;23:943-50.

[23] Craig CL, Marshall AL, Sjostrom M, Bauman AE, Booth ML, Ainsworth BE, et al. International physical activity questionnaire: 12 -country reliability and validity. Med Sci Sports Exerc 2003;35:1381-95.

[24] IPAQ. International Physical Activity Questionnaires. www.ipaq.ki.se/ipaq.htm [accessed 25.09.09].

[25] Deci EL, Ryan RM. Intrinsic motivation and selfdetermination in human behavior. New York: Plenum; 1985

[26] Laine C, Davidoff F. Patient-centered medicine. A professional evolution. JAMA 1996;275:152-6.

[27] Williams GC, Freedman ZR, Deci EL. Supporting autonomy to motivate patients with diabetes for glucose control. Diabetes Care 1998;21:1644-51.

[28] Williams GC, Grow VM, Freedman ZR, Ryan RM, Deci EL. Motivational predictors of weight loss and weight-loss maintenance. J Pers Soc Psychol 1996;70:115-26.

[29] Van der Bijl J, Van Poelgeest-Eeltink A, Shortridge-Baggett LM. The psychometric properties of the diabetes management self-efficacy scale for patients with type 2 diabetes mellitus. J Adv Nurs 1999;30:352-9.

[30] Halfens R. Locus of control: beheersingsorientatie in relatie tot ziekte en gezondheidsgedrag. Limburg University; 1985.

[31] Wallston KA, Wallston BS, DeVellis R. Development of the Multidimensional Health Locus of Control (MHLC) Scales. Health Educ Monogr 1978;6:160-70.

[32] Wallston KA, Stein MJ, Smith CA. Form C of the MHLC scales: a condition-specific measure of locus of control. J Pers Assess 1994;63:534-53.

[33] Williams GC, Rodin GC, Ryan RM, Grolnick WS, Deci EL. Autonomous regulation and long-term medication adherence in adult outpatients. Health Psychol 1998;17:269-76.

[34] Snoek FJ, Pouwer F, Welch GW, Polonsky WH. Diabetesrelated emotional distress in Dutch and U.S. diabetic patients: cross-cultural validity of the problem areas in diabetes scale. Diabetes Care 2000;23:1305-9.
[35] Rasbash J, Steele F, Browne W, Prosser B. A user's guide to Mlwin, version 2. Bristol: Centre for Multilevel Modelling, University of Bristol; 2005.

[36] Snijders TAB, Bosker RJ. Multilevel analysis: an introduction to basic and advanced multilevel modeling. London: Sage Publications Ltd; 1999.

[37] Surgenor LJ, Horn J, Hudson SM, Lunt H, Tennent J. Metabolic control and psychological sense of control in women with diabetes mellitus. Alternative considerations of the relationship. J Psychosom Res 2000;49:267-73.

[38] Watkins KW, Connell CM, Fitzgerald JT, Klem L, Hickey T, Ingersoll Dayton B. Effect of adults' self-regulation of diabetes on quality-of-life outcomes. Diabetes Care 2000;23:1511-5.

[39] Smith DE, Heckemeyer CM, Kratt PP, Mason DA. Motivational interviewing to improve adherence to a behavioral weight-control program for older obese women with NIDDM. A pilot study. Diabetes Care 1997;20:52-4.

[40] Smith-West D, DiLillo V, Bursac Z, Gore SA, Greene PG. Motivational interviewing improves weight loss in women with type 2 diabetes. Diabetes Care 2007;30:1081-7.

[41] Clark M, Hampson SE, Avery L, Simpson R. Effects of a tailored lifestyle self-management intervention in patients with type 2 diabetes. Br J Health Psychol 2004;9:365-79.

[42] Clark M, Hampson SE, Avery L, Simpson R. Effects of a brief tailored intervention on the process and predictors of lifestyle behaviour change in patients with type 2 diabetes. Psychol Health Med 2004;9:440-9.

[43] Ismail K, Thomas SM, Maissi E, Chalder T, Schmidt U, Bartlett $J$, et al. Motivational enhancement therapy with and without cognitive behavior therapy to treat type 1 diabetes: a randomized trial. Ann Intern Med 2008;149:708-19.

[44] Rubak S, Sandbaek A, Lauritzen T, Christensen B. Motivational interviewing: a systematic review and metaanalysis. Br J Gen Pract 2005;55:305-12.

[45] Emmons KM, Rollnick S. Motivational interviewing in health care settings. Opportunities and limitations. Am J Prev Med 2001;20:68-74.

[46] Brug J, Spikmans F, Aartsen C, Breedveld B, Bes R, Fereira I. Training dietitians in basic motivational interviewing skills results in changes in their counseling style and in lower saturated fat intakes in their patients. J Nutr Educ Behav 2007;39:8-12.

[47] Basch CE, Sliepcevich EM, Gold RS, Duncan DF, Kolbe LJ. Avoiding type III errors in health education program evaluations: a case study. Health Educ Q 1985;12:315-31.

[48] Rollnick S. Enthusiasm, quick fixes and premature controlled trials. Addiction 2001;96:1769-70.

[49] Madson MB, Loignon AC, Lane C. Training in motivational interviewing: a systematic review. J Subst Abuse Treat 2009;36:101-9.

[50] De Hollander AEM, Hoeymans N, Melse JM, Van Oers JAM, Polder JJ. Zorg voor gezondheid. Volksgezondheid Toekomst Verkenning 2006. RIVM-rapport nr. 270061003. Bohn Sfafleu Van Loghum, Houten; 2006.

[51] Keers JC, Bouma J, Links TP, ter Maaten JC, Gans RO, Wolffenbuttel BH, et al. One-year follow-up effects of diabetes rehabilitation for patients with prolonged selfmanagement difficulties. Patient Educ Couns 2006;60:16-23.

[52] Miller WR. When is it motivational interviewing? Addiction 2001;96:1770-1.

[53] Bartholomew LK, Parcel GS, Kok G, Gottlieb NH. Planning health promotion programs. An intervention mapping approach. San Francisco: Jossey-Bass; 2000. pp. 496-499. 